\title{
Corrigendum
}

\section{Corrigendum to "Slip Effect on MHD Chemically Reacting Convictive Boundary Layer Flow with Heat Absorption"}

\author{
Mekonnen Shifferaw Ayano $\mathbb{D}^{1}$ and Negussie Tadege Demeke ${ }^{2}$ \\ ${ }^{1}$ Department of Mathematics, University of Swaziland, P. Bag 4, Kwaluseni M201, Swaziland \\ ${ }^{2}$ Mechanical Engineering Department, Arba Minch University, B.O. Box 21, Arba Minch, Ethiopia \\ Correspondence should be addressed to Mekonnen Shifferaw Ayano; mekk_aya@yahoo.com
}

Received 9 August 2017; Accepted 24 August 2017; Published 1 January 2018

Copyright (C) 2018 Mekonnen Shifferaw Ayano and Negussie Tadege Demeke. This is an open access article distributed under the Creative Commons Attribution License, which permits unrestricted use, distribution, and reproduction in any medium, provided the original work is properly cited.

In the article titled "Slip Effect on MHD Chemically Reacting Convictive Boundary Layer Flow with Heat Absorption" [1] there were two errors, which should be corrected as follows:

(1) The horizontal axis in Figures 1 and 2 did not include sufficiently large values; corrected figures are presented below.

(2) The boundary condition at $y=0$, in (1), is rephrased (for clarity) in the form

$$
\begin{aligned}
& u=B x+L \frac{\partial u}{\partial y}, \\
& v=v_{w}, \quad w=0, \\
& \bar{\omega}=-n \frac{\partial u}{\partial y} \\
& T=T_{w}+E x^{r}, \\
& C=C_{w}+F x^{p},
\end{aligned}
$$

$$
\text { at } y=0 \text {, }
$$

where $B, E, F, r$, and $p$ are constants. follows:

The nondimensional variables (see (2)) are rephrased as

$$
\begin{aligned}
& \Psi=\sqrt{B v} x f(\eta), \\
& u=\frac{\partial \Psi}{\partial y}=B x f^{\prime(\eta)}, \\
& v=-\frac{\partial \Psi}{\partial x}=-\sqrt{B v} f(\eta),
\end{aligned}
$$

$$
\begin{aligned}
\eta & =y \sqrt{\frac{B}{v}}, \\
w & =B \times h(\eta), \\
\bar{\omega} & =\sqrt{\frac{B^{3}}{v}} x g(\eta) .
\end{aligned}
$$

Equations (3) will take the form

$$
\begin{aligned}
& f^{\prime \prime \prime}+f f^{\prime \prime}-\left(f^{\prime}\right)^{2}+N_{1} g^{\prime}-\frac{2 M}{\alpha_{e}^{2}+\beta_{e}^{2}}\left(\alpha_{e} f^{\prime}+B_{e} h\right) \\
& \quad=0, \\
& h^{\prime \prime}+h^{\prime} f-f^{\prime} h+\frac{2 M}{\alpha_{e}^{2}+\beta_{e}^{2}}\left(\beta_{e} f^{\prime}-\alpha_{e} h\right)=0, \\
& \lambda g^{\prime \prime}-\left(2 g+f^{\prime \prime}\right)=0, \\
& \frac{3 \operatorname{Ra}+r}{3 \operatorname{Pr}+\operatorname{Ra}} \theta^{\prime \prime}+f \theta^{\prime}-f^{\prime} \theta+A \theta+E c\left[\left(f^{\prime}\right)^{2}+\left(h^{\prime}\right)^{2}\right] \\
& \quad=0, \\
& \frac{1}{S c} \phi^{\prime \prime}+f \phi^{\prime}-f^{\prime} \phi-K \phi=0,
\end{aligned}
$$

where $\lambda=\omega B / \kappa v$ is micropolar parameter, $A=Q / C_{p} \rho B$ is internal heat generation/absorption parameter, $\operatorname{Pr}=v / \alpha$ is the Prandtl number, $M=\sigma B_{0}^{2} / \rho B$ is magnetic parameter, and $U_{0}=B x$. 


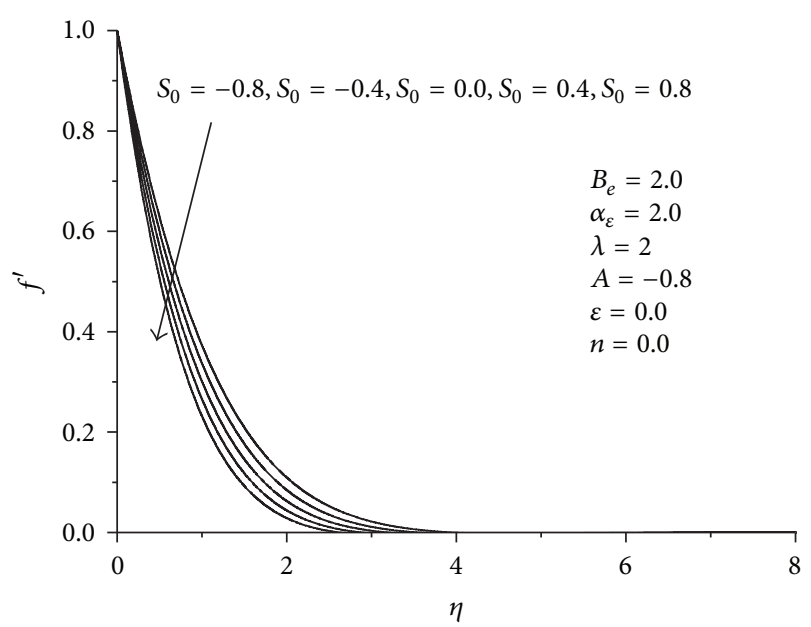

Figure 1: The effect of suction/injection on $f^{\prime}$.

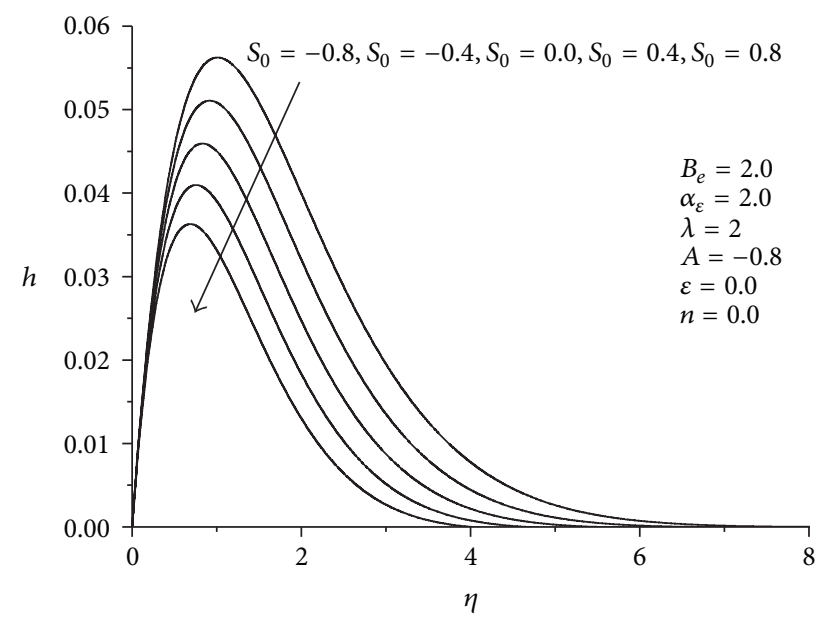

FIgURE 2: The effect of suction/injection on $h$.

\section{References}

[1] M. S. Ayano and N. T. Demeke, "Slip Effect on MHD Chemically Reacting Convictive Boundary Layer Flow with Heat Absorption," Journal of Engineering (United States), vol. 2016, Article ID 2689493, 9 pages, 2016. 


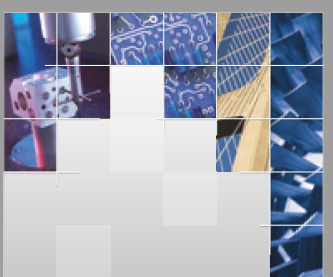

\section{Enfincering}
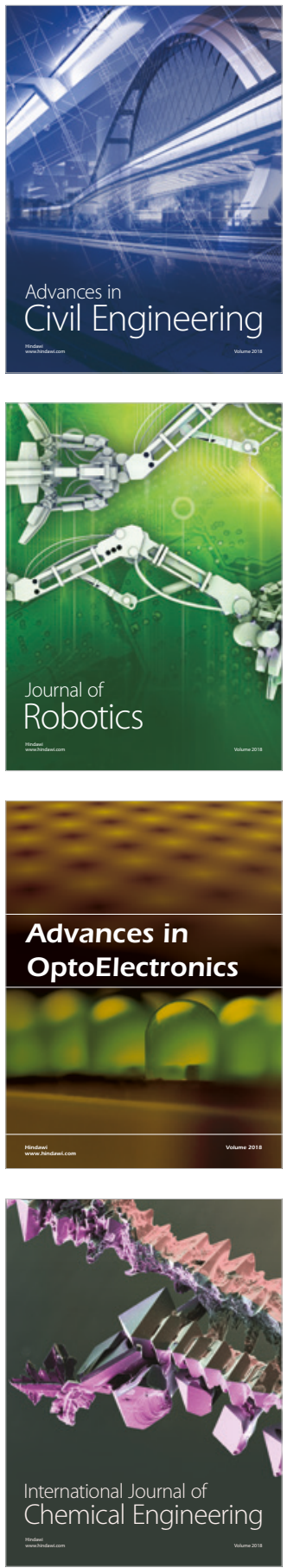

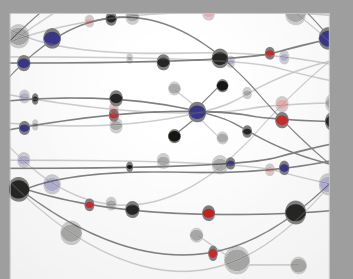

\section{Rotating \\ Machinery}

The Scientific World Journal

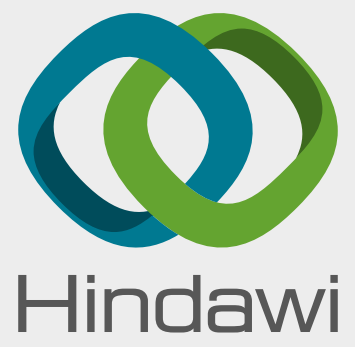

Submit your manuscripts at

www.hindawi.com
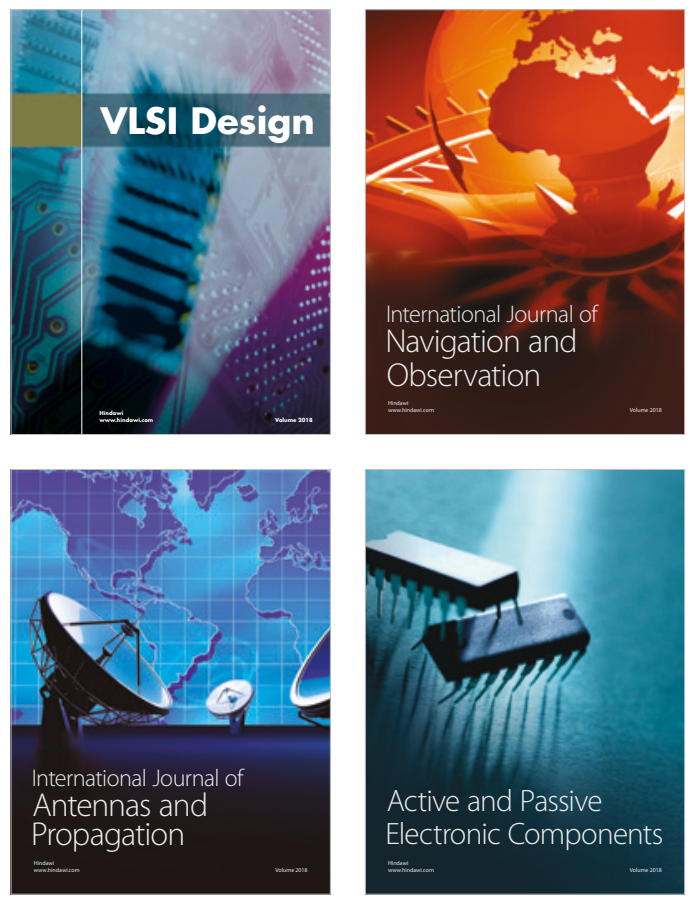
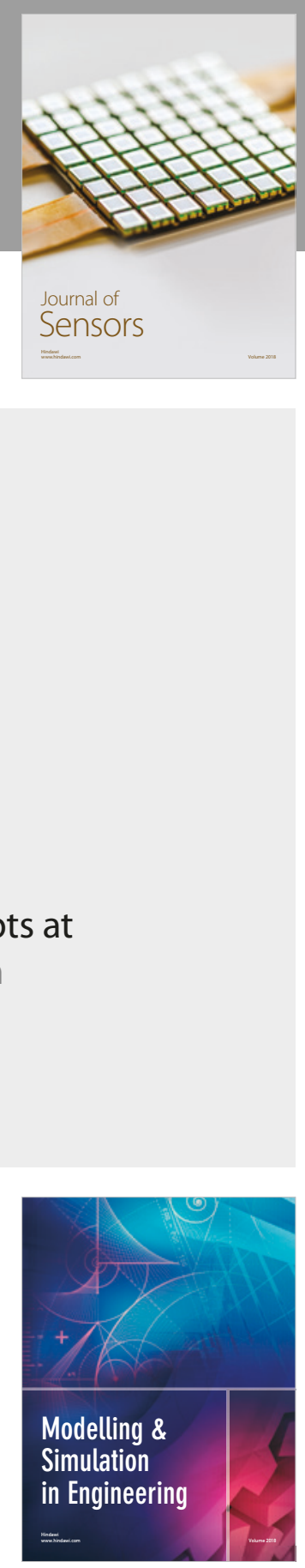

\section{Advances \\ Multimedia}
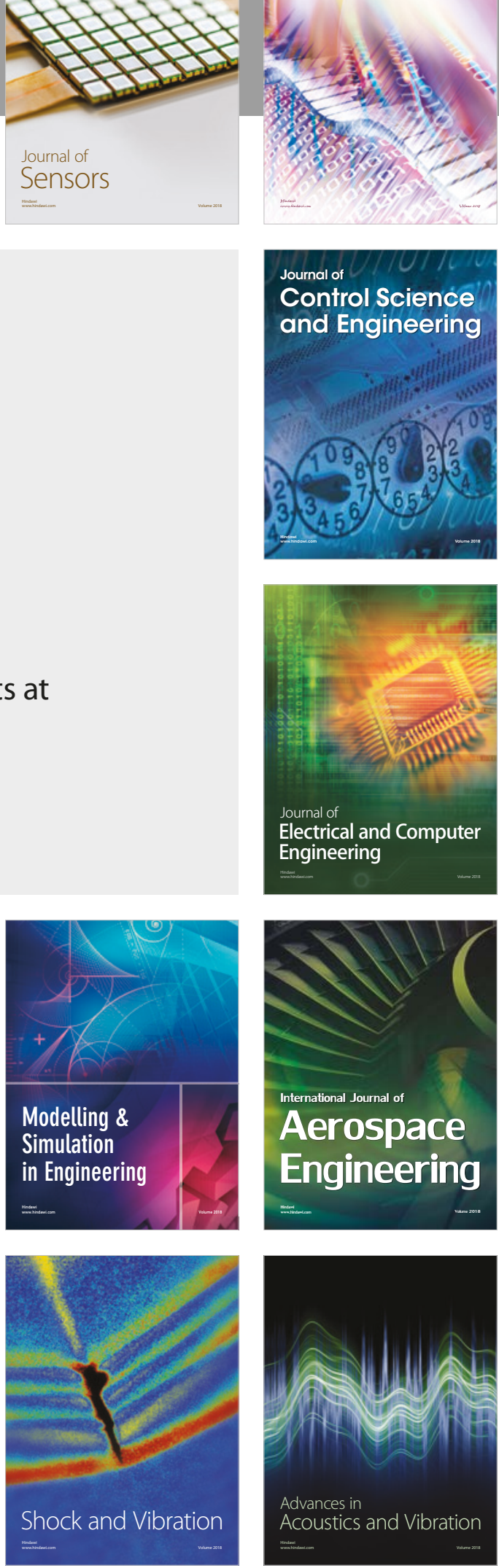